OPEN ACCESS

Edited by: Martin Heinze,

Immanuel Klinik Rüdersdorf be Berlin, Germany

Reviewed by:

Taiwo Lateef Sheikh, Federal Neuropsychiatric Hospital, Nigeria Andres Ricardo Schneeberger, Psychiatrische Dienste Graubuenden, Switzerland

*Correspondence: Ana Luiza Zaninotto azaninotto@neuromodulationlab.org

Specialty section:

This article was submitted to Public Mental Health, a section of the journal Frontiers in Psychiatry

Received: 03 February 2016 Accepted: 19 May 2016 Published: 14 June 2016

Citation:

Zaninotto AL, Vicentini JE, Fregni F

Rodrigues PA, Botelho C, de Lucia MCS and Paiva WS (2016) Updates and Current Perspectives of Psychiatric Assessments after Traumatic Brain Injury:

A Systematic Review.

Front. Psychiatry 7:95 doi: 10.3389/fpsyt.2016.00095

\section{Updates and Current Perspectives of Psychiatric Assessments after Traumatic Brain Injury: A Systematic Review}

\author{
Ana Luiza Zaninotto ${ }^{1,2 *}$, Jessica Elias Vicentini ${ }^{3}$, Felipe Fregni', Priscila Aparecida \\ Rodrigues $^{2}$, Cibele Botelho ${ }^{2}$, Mara Cristina Souza de Lucia ${ }^{4}$ and Wellingson Silva Paiva ${ }^{2}$ \\ 'Laboratory of Neuromodulation, Center for Clinical Research Learning, Harvard Medical School (HMS), Charlestown, MA, \\ USA, ${ }^{2}$ Department of Neurology, School of Medicine, University São Paulo (USP-SP), São Paulo, Brazil, ${ }^{3}$ Department of \\ Neurology, School of Medical Sciences, State University of Campinas (UNICAMP), Campinas, Brazil, ${ }^{4}$ Division of Psychology, \\ School of Medicine, University São Paulo (USP-SP), São Paulo, Brazil
}

Neuropsychological and psychiatric disorders represent a major concern and cause of disabilities after the trauma, contributing to worse recovery after traumatic brain injury (TBI). However, the lack of well-defined parameters to evaluate patient's psychiatric disorders leads to a wide range of diagnoses and symptoms. The aim of this study was to perform a review of literature in order to gather data of the most common scales and inventories used to assess and diagnose depression, anxiety, and posttraumatic stress disorder (PTSD) after TBI. We conducted a literature search via MEDLINE, PubMed, and Web of Science. We included reviews, systematic reviews, and meta-analysis studies, and we used the following keywords: "traumatic brain injury OR TBI," "depression OR depressive disorder," "anxiety," and "posttraumatic stress disorder OR PTSD." From 610 titles, a total of 68 systematic reviews or meta-analysis were included in the section "Results" of this review: depression ( $n=32)$, anxiety $(n=9)$, and PTSD $(n=27)$. Depression after TBI is a more established condition, with more homogeneous studies. Anxiety and PTSD disorders have been studied in a heterogeneous way, usually as comorbidity with other psychiatric disorders. Some scales and inventories designed for the general community may not be appropriate for patients with TBI.

Keywords: traumatic brain injury, psychiatry, depression, anxiety, posttraumatic stress disorder, assessment, scales, inventory reporting, questionnaires

\section{INTRODUCTION}

Globally, traumatic injuries are responsible for more than five million deaths annually, and traumatic brain injury (TBI) is one of the leading causes of disabilities and death. It is estimated that 1.7 million cases of TBI occur each year in the United States (USA), resulting in 52,000 deaths (1). Therefore, TBI represents around one-third (30.5\%) of all injury-related deaths in the USA (1).

Traumatic brain injury usually results in brain disorders, leading to a heterogeneous spectrum of morbidities, ranging from transitory disturbances to permanent symptoms (2-6). Cognitive and psychiatric disorders are the common causes of disabilities and may cause difficulties in recovery after TBI (7-10). In diffuse axonal injury, disruption of the neural circuitry between the prefrontal 
cortex and limbic system $(11,12)$ can result in mood disorders arising even weeks or months after the initial injury (13).

Gordon et al. (14) reviewed rehabilitation in TBI and highlighted the need for a better understanding of the dynamics of recovery. They argue that only a few studies used measures accepted as "gold standards" (14). In 2010, the National Institute of Neurological Disorders and Stroke (NINDS) Common Data Elements (CDE) was created to develop data standards for clinical research in patients with TBI (15). However, even with the proposal of some guidelines, psychiatric functions are still being assessed in a heterogeneous manner $(16,17)$. Therefore, the aim of this study is to summarize the literature, including reviews, systematic reviews, and meta-analyses, regarding the scales and inventories most commonly used to diagnose and evaluate depression, anxiety, and posttraumatic stress disorder (PTSD) in patients with TBI. To assess a large number of published articles, we used an original method in order to have a global view of the instruments used in diagnosis over the years.

\section{METHODS}

We conducted a literature search via online databases including MEDLINE, PubMed, and Web of Science. We included reviews, systematic reviews, and meta-analysis studies. In our search, we used the following keywords: "traumatic brain injury OR TBI," "depression OR depressive disorder," "anxiety," and "posttraumatic stress disorder OR PTSD." Abstracts and full text were carefully read, and studies were included in our review if they fulfilled the following inclusion criteria: (a) description/citation of the scale, questionnaire, or inventory used, (b) published in a peer-reviewed journal, (c) description of quantitative assessment for diagnosis, (d) full text written in English, and (e) adult participants. We selected studies published up to February 2016.

Searching and data analysis were performed by Ana Luiza Zaninotto and Jessica Elias Vicentini, both of whom have experience with mental health intervention and clinical research in TBI. All reviews and full text were read by the two reviewers and were included if they met the above-mentioned inclusion criteria. This selection method follows previous literature (18).

\section{RESULTS}

We reviewed 610 titles and abstracts and selected studies according to our inclusion and exclusion criteria. Of those, 362 studies were excluded, of which 248 were reviewed entirely (full text). Sixty-nine studies were included in the review focusing on one or more aspects of the following three topics of interest: (a) depression $(n=32)$, (b) anxiety $(n=9)$, and (c) PTSD $(n=27)$. Of the 68 studies, 11 studies had overlapping topics of interest, since they met criteria for more than one psychiatric disorder (17-27). The abstracts and full text that did not meet the inclusion criteria were excluded from the review $(n=541)$. The main reasons for exclusion were that the studies did not report the instruments used to assess the psychiatric disorders and/or the psychiatric assessment was not the center of the study $(n=447)$. The remaining excluded articles $(n=94)$ were either not related to TBI samples, focused on the neurological basis of the psychiatric disease, discussed pharmacological interventions, or did not focus on psychiatric disorders (depression, anxiety, or PTSD).

Each step of the search and review process is detailed in a flow diagram (Figure 1), based on the PRISMA work group (28).

In Table 1, we present studies $(n=31)$ that assessed depressive symptoms or diagnosed depression following TBI. Five of these were meta-analyses. Seven studies analyzed assessments of depression related to TBI in veterans, military personnel, or warrelated injuries. We observed that the Beck Depression Inventory (BDI) was presented in all the studies, followed by Structured Clinical Interview for DSM (SCID) and Diagnostic and Statistical Manual (DSM) diagnosis criteria. For TBI populations, the BDI, Symptoms Checklist (SCL), and Center for Epidemiologic Studies-Depression Scale (CES-D) were the most cited selfreported scales. For diagnosis of depression, DSM criteria were the most commonly used, followed by International Classification of Diseases (ICD). The most commonly used instrument was the Hamilton Depression Rating Scale (HAM-D). Table 2 shows different instruments were cited to assess depression.

Table 3 shows the nine studies that assessed anxiety disorders after TBI. Eight of these studies overlapped with other psychiatric conditions. Just one review focused on the anxiety sequelae after TBI (99). The Hospital Anxiety and Depressive Symptoms Scale (HADS) was the most cited instrument to assess anxiety, followed by State-Trait Anxiety Inventory (STAI). The DSM criteria were most commonly used to diagnose anxiety. Table 4 shows the instruments cited in the anxiety reviews that were analyzed.

In our search, we found 26 reviews and meta-analyses related to PTSD and TBI. We identified two types of studies, one focusing on military veterans or war-related TBI $(n=13)$, and another focusing on a non-specific TBI population $(n=13)$ (Table 5). Table 6 shows a summary of the scales and inventories used to assess PTSD in TBI populations. The PTSD Checklist (PCL) is most commonly used to assess PTSD in veteran and military samples, followed by the Clinician-Administered PTSD Scale (CAPS). PTSD Checklist - Military version (PCL-M) and PTSD Checklist - Civilian version (PCL-C) were the most cited selfreported scales. DSM criteria were used to diagnose PTSD, while ICD was not cited in any of the studies we analyzed.

\section{DISCUSSION}

\section{Depression}

Major depression and dysthymia are frequently diagnosed using structured clinical interviews meeting DSM or ICD criteria. Depression often occurs in the first year after TBI (12). Estimates for posttraumatic depression range from 6 to $77 \%(151,152)$, depending on diagnostic criteria, assessment methods, and timing post-trauma $(22,23,152)$. Concomitant brain injury is a strong predictor of depression after TBI (13). In addition, poor mental health after TBI involves several factors, including young age at the time of injury, short duration between the injury and assessment, pain, lower levels of social support (153), and lack of hope (40). For those patients, consequences of depression include greater interpersonal difficulties, higher rates of unemployment (152), increased rates of distress, and problems with rehabilitation (154). 


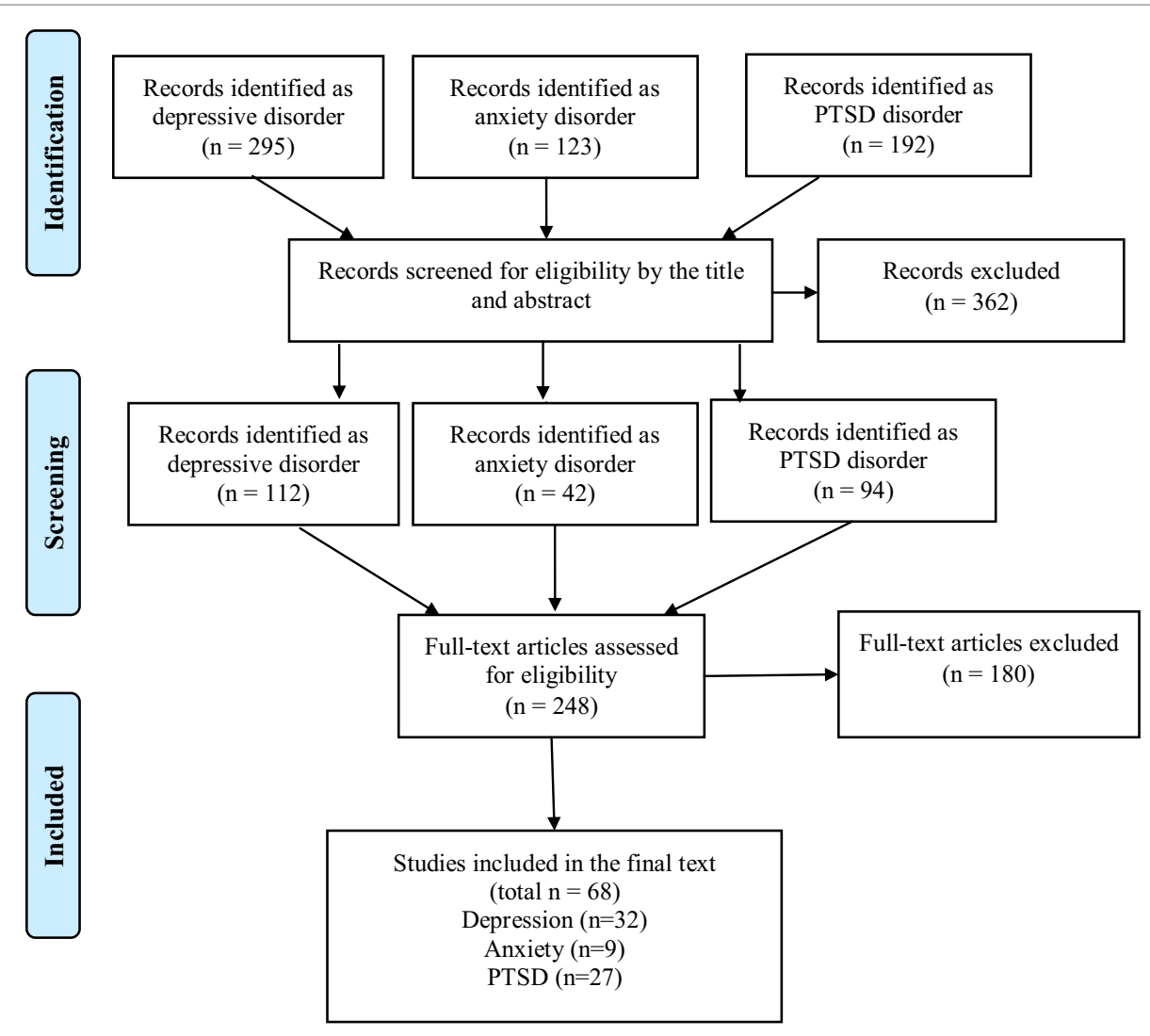

FIGURE 1 | Flow diagram

Some studies focus on specific TBI populations including the elderly, women, and veterans. Menzel (38) reviewed depression in the elderly after TBI, but the author found only one original study (104), leading to inconclusive findings. In their study, Levin et al. (104) assessed depressive symptoms by the Geriatric Depression Scale (GDS). Since this scale has been designed and standardized for geriatric populations with no history of TBI, there is a potential risk of overlapping the symptoms of TBI and depression. In addition, GDS cannot be used as a criterion for diagnostic assessment.

Seven studies addressed combat veterans with sustained TBI $(18,19,22,23,31,33,36)$. They reported limited evidence that deficits and symptoms are distinct between veterans with or without a history of mild TBI (mTBI). O'Neil et al. (23) also highlighted a study (155) that showed an increased risk of suicide post-TBI compared to the non-TBI population. As we reported before, the BDI was the most cited inventory used in this sample. It contains 21 symptoms correlated with selfreported depression. The newer version of the BDI, the Beck Depression Inventory II (BDI-II), produced scores two points higher when compared to the oldest version for psychiatric outpatients (49). For this reason, comparisons between studies need to be carefully done.

One study addressed the literature focusing on women with TBI, comorbidity with depression, and hopelessness (40). The study analyzed symptoms both qualitatively and quantitatively. They concluded that mental health seems to deteriorate after TBI. Social isolation is of particular concern as a consequence of poor emotional functioning in these patients. In their study, the authors also reported on sex-based differences and limited data on the incidence of sex-specific depression.

Osborn et al. (17) showed the prevalence of major depression disorder (MDD) and dysthymia ranged from 14\% using International Classification o Diseases (ICD-10) criteria to $43 \%$ using DSM-III criteria. For self-reported scales, the range of depression was between 16 and 33\%. They found higher prevalence rates of depression using NFI than SCID-I, Schedules for Clinical Assessment in Neuropsychiatry (SCAN), or MiniInternational Neuropsychiatric Interview (MINI). The occurrence of MDD and dysthymia appears to rise in the first 5 years after brain injury (from 21 to $43 \%$ ). However, the majority of the studies used mixed TBI severity samples and did not report separate outcomes for these subgroups (17). Still, the HAM-D is wildly used to diagnose MDD in patients with TBI.

It is appropriate to use the standard diagnostic criteria for depression when evaluating persons with TBI. The CDE recommends scales and inventories to assess symptoms of depression in adults with TBI (15): the BDI-II, Brief Symptom Inventory-18 (BSI-18), CES-D, and Patient Health Questionnaire-9-Item (PHQ-9). 
TABLE 1 | Characteristics of the studies and the scales and inventories used to assess depressive disorder after TBI.

\begin{tabular}{|c|c|c|c|c|}
\hline Reference & Study design & Study population & $\begin{array}{l}\text { Number of } \\
\text { analyzed studies/ } \\
\text { total of studies }\end{array}$ & Instruments \\
\hline Adamson et al. (29) & Meta-analysis & Neurologic patients & $(3 / 26)$ & BDI, HADS \\
\hline Barker-Collo et al. (30) & Meta-analysis & TBI & 13 & BDI, BDI-II, CES-D, HAM-D, LSSAD, SCL-90-R \\
\hline Capehart and Bass (19) & Review & Veterans with TBI and PTSD & N/A & HAM-D, BDI \\
\hline Cooper et al. (31) & Systematic review & Military veterans with mTBI & $(4 / 19)$ & BDI, BDI-II \\
\hline Crisp (32) & Systematic review & MDD, SCI, TBI, CBP, MI/CG & $(8 / 54)$ & BDI, GHQ, CES-D, HAM-D, SCL-90-R \\
\hline Daggett et al. (33) & Systematic review & Combat veterans who had sustained TBI & $(3 / 17)$ & BDI, CES-D, CPRS, SCID, VAS-D \\
\hline Fann et al. (34) & Systematic review & $\mathrm{TBI}$ & 26 & $\begin{array}{l}\text { BDI, BDI-II, BPRS, DSM-III-R, HADS, HAM-D, } \\
\text { MADRS, PHQ-9, SCL-90-R }\end{array}$ \\
\hline Fleminger et al. (35) & Review & TBI & $(9 / \mathrm{N} / \mathrm{A})$ & BDI, DSM-IV, HAM-D, NFI, SCL-R-90 \\
\hline Garrelfs et al. (20) & Systematic review & $\mathrm{ABI}$ & $(6 / 7)$ & BDI, HADS, HAM-D, SCID-I \\
\hline Gordon et al. (14) & Systematic review & $\mathrm{TBI}$ & N/A & $\begin{array}{l}\text { BDI, BDI-II, DSM-IV, CES-D, MCMI, MMPI II, NFI } \\
\text { SCL-90-R, SCID }\end{array}$ \\
\hline Halbauer et al. (36) & Review & War-related mild to moderate TBI & N/A & BDI, DSM-III, DSM-III-R, DSM-IV-TR, NFI \\
\hline Hesdorffer et al. (37) & Systematic review & $\mathrm{TBI}$ & $(4 / N / A)$ & ICD-9-CM; DIS; PSE, SCID \\
\hline Kim et al. (21) & Systematic review & $\mathrm{TBI}$ & $(15 / 66)$ & $\begin{array}{l}\text { BDI, BDI-II, SCID, DSM III, DSM-III-R, DSM-IV, CES-D, } \\
\text { NFI, NIMH-DIS, mNIMH-DIS, SCID-I, Wimbledon-SRS }\end{array}$ \\
\hline Matarazzo et al. (18) & Systematic review & Veterans with TBI & 3 & BDI-II, SCID \\
\hline Menzel (38) & Systematic review & TBI in elderly & 1 & GDS \\
\hline Nowrangi et al. (39) & Review & TBI with suicidal risk & N/A & BDI, HAM-D, SCID \\
\hline O'Neil et al. (22) & Systematic review & Veterans/military population with mTBI & $(8 / 31)$ & BDI-II, DSM-IV, HADS, SCID-I \\
\hline O’Neil et al. (23) & Systematic review & Veterans/military population with mTBI & $(8 / 31)$ & BDI-II, DSM-IV, HADS, SCID-I \\
\hline Osborn et al. (17) & Meta-analysis & Closed TBI & 93 & $\begin{array}{l}\text { BDI-II, BDICES-D, CIDI, CIS, DIS, DSM-III, DSM-IV, } \\
\text { GDS, HADS, ICD-10, LSSAD, MADRS, MINI, SCAN, } \\
\text { SCID, PSE, PHQ-9, NFI, SADS-L, SCID, ZSDS }\end{array}$ \\
\hline Oyesanya and Ward (40) & Systematic review & Woman with $\mathrm{TBI}$ & 12 & BDI-II, Adaptation of BRFSS, CES-D, HADS, DSM-IV \\
\hline Panayiotou et al. (24) & Meta-analysis & $\mathrm{mTBI}$ & $(9 / 11)$ & $\begin{array}{l}\text { BDI, CES-D, HAM-D, NBAP, POMS, SCL-90, } \\
\text { SCL-90-R, ZSDS }\end{array}$ \\
\hline Rogers and Read (25) & Systematic review & $\mathrm{TBI}$ & $(13 / N / A)$ & $\begin{array}{l}\text { CES-D, CID, CID-9-CM, DSM-III, DSM-IV, DIS, } \\
\text { HAM-D, MMPI, NFI, PSE, PTSD-I, SCAN, SCL-90-R }\end{array}$ \\
\hline Rosenthal et al. (41) & Systematic review & $\mathrm{TBI}$ & 30 & $\begin{array}{l}\text { BDI, BPRS, CAQ, DSM-III, DSM-III-R, LSSAD, HSCL, } \\
\text { HAM-D, MMPI, NIMH-DIS, PACL, PAI, PSE, POMS, } \\
\text { SCL-90-R, ZSDS }\end{array}$ \\
\hline Sherer et al. (42) & Systematic review & $\mathrm{TBl}$ & 23 & BDI, HADS, NEO-PI-R, Wimbledon-SRS \\
\hline Simpson and Tate (43) & Review & TBI & 19 & BDI, BHS, DSM-III-R, PSE \\
\hline Soo and Tate (26) & Systematic review & mTBI & 3 & BDI, SCL-90-R \\
\hline Stalder-Luthy et al. (44) & Meta-analysis & $\mathrm{ABI}$ & 13 & $\begin{array}{l}\text { BDI, BDI-II, CIQ, CES-D, CSA, DASS-21, DDS, ERS, } \\
\text { GAS, GSI, HADS, MHLC, POMS, PSS, PHQ-9, RSE, } \\
\text { SCL-90, SIP, TSK }\end{array}$ \\
\hline Steel et al. (45) & Systematic review & Traumatic injury & N/A & $\begin{array}{l}\text { BDI, BSI-18, CES-D, DISCs, HADS, HAM-D, } \\
\text { HSCL-20, NFI, PHQ-9, SCID, ZSDS }\end{array}$ \\
\hline Vahle et al. (46) & Review & People with disabilities & $(7 / \mathrm{N} / \mathrm{A})$ & BDI, BSI, CES-D, DACL, MEDS, TBDI, ZSDS \\
\hline van Velzen et al. (47) & Systematic review & $\begin{array}{l}\text { Traumatic and } \\
\text { non-traumatic } \mathrm{ABI}\end{array}$ & 22 & BDI, NFI, SCID \\
\hline Warden et al. (27) & Systematic review & TBI & $(7 / 14)$ & BDI, HAM-D, DSM-IV \\
\hline
\end{tabular}

ABI, acquired brain injury; BDI, Beck Depression Inventory 2nd edition; BDI, Beck Depression Inventory; BHS, Beck Hopelessness Scale; BPRS, Brief Psychiatric Ranting Scale; BRFSS, Behavioral Risk Factor Surveillance System; BSI, Brief Symptom Inventory; BSI-18, Brief Symptom Inventory-18; CAQ, Clinical Analysis Questionnaire; CBP, chronic back pain; CES-D, Center for epidemiological studies; CIDI, Composite International Diagnostic Interview; CIS, Clinical Interview Schedule; CPRS, Compreensive Psychopathological Rating Scale; DACL, Depression Adjective Checklist; DASS-21, Depression Anxiety Stress Scale, DIS, Diagnostic Interview Scale; DISCs, Depression Intensity Scale Circles; DSM-III, Diagnostic and Statistical Manual 3rd edition; DSM-IV, Diagnostic and Statistical Manual 4th edition; GDS, Geriatric Depression Scale; GHQ, General Health Questionnaire; HADS, Hospital Anxiety and Depression Scale; HAM-D, Hamilton Depression Rating Scale; HSCL, Hopkins Symptom Checklist; HSCL-20, Hopkins Symptom Checklist-20; ICD- 10, International Classification of Diseases 10th revision; ICD-9, International Classification of Diseases 9th revision; LSSAD, Leeds Scale for the self-assessment of Anxiety and Depression; MADRS, Montgomery-Asberd Depression Rating Scale; MCMI, Millon Clinical Multiaxial Inventory; MDD, Major depressive disorder; MEDS, Medical-Based Emotional Distress Scale; MINI, Mini-International Neuropsychiatric Interview; MI/CG, myocardial infarction/coronary bypass grafting; MMPI, Minnesota Multiphasic Personality Inventory; MMPI-2, Minnesota Multiphasic Personality Inventory 2; mNIMH-DIS, Modified NIMH's Diagnostic Interview Schedule; mTBI, mild traumatic brain injury; NEO-PI-R, NEO Personality Inventory Revised; NFI, Neurobehavioral Functioning Inventory; NIMH-DIS, NIMH's Diagnostic Interview Schedule; PACL, Personality Adjective Checklist; PAI, Portland Adaptability Inventory; PHQ-9, Patient Health Questionnaire-9; POMS, Profile of Mood State; PSE, Present State Examination; SADS-L, Schedule for Affective Disorders and Schizophrenia (lifetime); SCAN, Schedules Clinical Assessment in Neuropsychiatry; SCI, spinal cord injury; SCID, Structured Clinical Interview for DSM; SCL 90-R, Symptoms Checklist 90-Revised; STAI, State-Trait Anxiety Inventory; TBDI, Talbieh Brief Distress Inventory; VAS-D, Visual Analog Scale for Depression; Wimbledon-SRS, Wimbledon Self-Report Scale; ZSDS, Zung Self-Rating Depression Scale. 
TABLE 2 | Scales and inventories used to assess depressive disorder after TBI.

\begin{tabular}{|c|c|c|}
\hline Name of the scale & Abbreviation & Clinical utility \\
\hline Beck Depression Inventory (48) & $\mathrm{BDI}$ & Interview schedule \\
\hline Beck Depression Inventory 2nd edition (49) & $\mathrm{BDI}-\|$ & Interview schedule \\
\hline Beck Hopelessness Scale (50) & $\mathrm{BHS}$ & Interview schedule \\
\hline Behavioral Risk Factor Surveillance System (51) & BRFSS & Interview schedule \\
\hline Brief Psychiatric Rating Scale (52) & BPRS & Interview schedule \\
\hline Brief Symptom Inventory (53) & $\mathrm{BSI}$ & Interview schedule \\
\hline Brief Symptom Inventory-18 (54) & BSI-18 & Interview schedule \\
\hline Center for Epidemiologic Studies - Depression form (55) & CES-D & Interview schedule \\
\hline Clinical Analysis Questionnaire (56) & $\mathrm{CAQ}$ & Interview schedule \\
\hline Clinical Interview Schedule (57) & CIS & Interview schedule \\
\hline Composite International Diagnostic Interview (58) & CIDI & Interview schedule \\
\hline Comprehensive Psychopathological Rating Scale (59) & CPRS & Interview schedule \\
\hline Depression Adjective Checklist (60) & DACL & Interview schedule \\
\hline Depression Anxiety Stress Scale 21 (61) & DASS-21 & Interview schedule \\
\hline Depression Intensity Scale Circles (62) & DISCs & Interview schedule \\
\hline Diagnostic and Statistical Manual 3rd edition [DSM-III (63)] & DSM-III & Diagnose \\
\hline Diagnostic and Statistical Manual 4th edition [DSM-IV (64)] & DSM-IV & Diagnose \\
\hline Diagnostic Interview Scale (65) & DIS & Interview schedule \\
\hline General Health Questionnaire (66) & $\mathrm{GHQ}$ & Interview schedule \\
\hline Geriatric Depression Scale (67) & GDS & Interview schedule \\
\hline Hamilton Depression Rating Scale (68) & HAM-D & Diagnose \\
\hline Hopkins Symptom Checklist (69) & HSCL & Interview schedule \\
\hline Hopkins Symptom Checklist-20 & HSCL-20 & Interview schedule \\
\hline Hospital Anxiety and Depression Scale (70) & HADS & Interview schedule \\
\hline International Classification of Diseases 9th revision (71) & ICD-9 & Diagnose \\
\hline International Classification of Diseases 10th revision (72) & ICD-10 & Diagnose \\
\hline Leeds Scale for the self-assessment of Anxiety and Depression (73) & LSSAD & Interview schedule \\
\hline Mayo-Portland Adaptability Inventory (74) & MPAl & Interview schedule \\
\hline Medical-Based Emotional Distress Scale (75) & MEDS & Interview schedule \\
\hline Millon Clinical Multiaxial Inventory (76) & $\mathrm{MCMl}$ & Interview schedule \\
\hline Mini-International Neuropsychiatric Interview (77) & $\mathrm{MINI}$ & Interview schedule \\
\hline Minnesota Multiphasic Personality Inventory - 2 (78) & MMPI-2 & Interview schedule \\
\hline Minnesota Multiphasic Personality Inventory (79) & MMPI & Interview schedule \\
\hline Montgomery-Asberg Depression Rating Scale (80) & MADRS & Diagnose \\
\hline NEO Personality Inventory Revised (81) & NEO-PI-R & Interview schedule \\
\hline Neurobehavioral Functioning Inventory (82) & NFI & Interview schedule \\
\hline NIMH’s Diagnostic Interview Schedule (83) & $\mathrm{NIMH}-\mathrm{DIS}$ & Diagnose \\
\hline NIMH's Diagnostic Interview Schedule modified (84) & mNIMH-DIS & Diagnose \\
\hline Patient Health Questionnaire-9 (85) & PHQ-9 & Interview schedule \\
\hline Personality Adjective Checklist (86) & PACL & Interview schedule \\
\hline Present State Examination (87) & PSE & Interview schedule \\
\hline Profile of Mood State (88) & POMS & Interview schedule \\
\hline Schedule for Affective Disorders and Schizophrenia (lifetime) (89) & SADS-L & Interview schedule \\
\hline Schedules Clinical Assessment in Neuropsychiatry (90) & SCAN & Interview schedule \\
\hline State-Trait Anxiety Inventory (91) & STAI & Interview schedule \\
\hline Structured Clinical Interview (92) & SCID & Diagnose \\
\hline Symptoms Checklist 90-Revised (93) & SCL 90-R & Interview schedule \\
\hline Talbieh Brief Distress Inventory (94) & TBDI & Interview schedule \\
\hline Visual Analog Scale for Depression $(95,96)$ & VAS-D & Interview schedule \\
\hline Wimbledon Self-Report Scale (97) & Wimbledon-SRS & Interview schedule \\
\hline Zung Self-Rating Depression Scale (98) & ZSDS & Diagnose \\
\hline
\end{tabular}

\section{Anxiety}

Anxiety disorders are frequently comorbid after TBI; there is a complex and multifaceted relationship, considering that premorbid anxiety is a predictor of the development of depression and anxiety disorders post-TBI (156).
Anxiety disorders post-TBI have multiple etiologies, from environmental to biological/genetic. Anxious reactions usually follow brain injury occurring in the setting of traumatic events, such as motor vehicle accidents, falls, and assaults (157). We observed in our results that just one study aimed to analyze the 
TABLE 3 | Characteristics of the studies and the scales and inventories used to assess anxiety disorder after TBI.

\begin{tabular}{|c|c|c|c|c|}
\hline Reference & Study design & Study population & $\begin{array}{l}\text { Number of analyzed } \\
\text { studies/total of studies }\end{array}$ & Instruments \\
\hline Garrelfs et al. (20) & Systematic review & $\mathrm{ABI}(\mathrm{TBI}=4)$ & $(3 / 7)$ & HADS, HAM-A, NRS, STAI \\
\hline Moore et al. (99) & Review & mTBI & N/A & BAI, MMPI, MCMI-III, STAI \\
\hline O'Neil et al. (22) & Systematic review & Veterans/military with mTBI & $(6 / 31)$ & HADS, NSI \\
\hline O’Neil et al. (23) & Systematic review & Veterans/military with mTBI & $(6 / 31)$ & HADS, NSI \\
\hline Osborn et al. (17) & Meta-analysis & Closed TBI & 41 & $\begin{array}{l}\text { BAI, HADS, DSM-IV; DSM-III-R, DSM-IV; ICD-10, LSSAD, } \\
\text { MINI, SCID-I, SCAN, SADS-L; STAI }\end{array}$ \\
\hline Panayiotou et al. (24) & Meta-analysis & $\mathrm{mTBI}$ & $(5 / 11)$ & BAI, CAPS, GHQ, HTQ, IES, POMS, SCL-90-R \\
\hline Rogers and Read (25) & Review & $\mathrm{TBI}$ & N/A & $\begin{array}{l}\text { BEC, BSQ, DES, DIS, DSM-III, MMPI, PCSSC, DSM-IV, SCAN, } \\
\text { SCL-90-R }\end{array}$ \\
\hline Soo and Tate (26) & Systematic review & mTBI & 3 & BAI, IES, SCL-90-R, STAI-S \\
\hline Warden et al. (27) & Systematic review & TBI & $(1 / 14)$ & Y-BOCS \\
\hline
\end{tabular}

$A B I$, acquired brain injury; $m T B I$, mild traumatic brain injury.

Scales: BAI, Beck Anxiety Inventory; BEC, Behavior Evaluation Checklist; BSQ, Body Sensations Questionnaire; DES, Dissociative Experience Scale; DIS, Diagnostic Interview Schedule; DSM-III, Diagnostic and Statistic Manual 3rd edition; DSM-IV, Diagnostic and Statistic Manual 4th edition; GHQ, General Health Questionnaire; HADS, Hospital Anxiety and Depression Scale; HAM-A, Hamilton Anxiety Scale; HTQ, Harvard Trauma Questionnaire; ICD-10, International Classification of Diseases; IES, Impact of Events Scale; LSSAD, Leeds Scale for the Self-Assessment of Anxiety and Depression; MCMI-III, Millon Clinical Multiaxial Inventory 3rd edition; MINI, Mini-International Neuropsychiatric Interview; MMPI, Minnesota Multiphasic Personality Inventory; NRS, Neurobehavioral Rating Scale; NSI, Neurobehavioral Symptom Inventory; PCSSC, Post-Concussion Syndrome Symptom Checklist; POMS, Profile of Mood State; PTSD-I, PTSD Interview; SADS, Schedule for Affective Disorders and Schizophrenia; SCAN, Schedules for Clinical Assessment in Neuropsychiatry; SCID-I, Structured Clinical Interview; SCL-90R, Symptoms Checklist, 90R; STAI, State-Trait Anxiety Inventory; Y-BOCS, Yale-Brown Obsessive Compulsive Scale.

TABLE 4 | Scales and inventories used to assess anxiety disorder after TBI.

\begin{tabular}{|c|c|c|}
\hline Name of the scale & Abbreviation & Clinical utility \\
\hline Beck Anxiety Inventory (50) & $\mathrm{BAI}$ & Interview schedule \\
\hline Diagnostic Interview Schedule (65) & DIS & Interview schedule \\
\hline Diagnostic and Statistic Manual 3rd edition [DSM-III (63)] & DSM-III & Diagnose \\
\hline Diagnostic and Statistic Manual 3rd edition-revised [DSM-III-R (100)] & DSM-III-R & Diagnose \\
\hline Diagnostic and Statistic Manual 4th edition [DSM-IV (64)] & DSM-IV & Diagnose \\
\hline General Health Questionnaire (66) & $\mathrm{GHQ}$ & Interview schedule \\
\hline Hamilton Anxiety Scale (68) & HAM-A & Diagnose \\
\hline Hospital Anxiety and Depression Scale (70) & HADS & Interview schedule \\
\hline Impact of Events Scale (101) & IES & Interview schedule \\
\hline International Classification of Disease (102) & ICD-10 & Diagnose \\
\hline Leeds Scale for the Self-Assessment of Anxiety and Depression (103) & LSSAD & Interview schedule \\
\hline Millon Clinical Multiaxial Inventory 3rd edition (76) & MCMI-III & Interview schedule \\
\hline Mini-International Neuropsychiatric Interview (77) & $\mathrm{MINI}$ & Interview schedule \\
\hline Minnesota Multiphasic Personality Inventory (79) & MMPI & Interview schedule \\
\hline Neurobehavioral Rating Scale-Revised (104) & NRS-R & Interview schedule \\
\hline Profile of Mood State (88) & POMS & Interview schedule \\
\hline Schedule for Affective Disorders and Schizophrenia (89) & SADS & Interview schedule \\
\hline Schedules for Clinical Assessment in Neuropsychiatry (90) & SCAN & Interview schedule \\
\hline State-Trait Anxiety Inventory (91) & STAl & Interview schedule \\
\hline Structured Clinical Interview (105) & SCID-I & Diagnose \\
\hline Symptoms Checklist - 90R (106) & SCL-90R & Interview schedule \\
\hline Yale-Brown Obsessive Compulsive Scale (107) & Y-BOCS & Interview schedule \\
\hline
\end{tabular}

sequelae of anxiety disorders after TBI (99). The other studies focused on the overall mental condition, including assessment of anxiety. One study analyzed the overall mental condition in a heterogeneous sample of patients with acquired brain injury (20). Three studies selected homogeneous samples: patients with closed TBI (17) and veterans/military personnel with $\operatorname{mTBI}(22,23)$.

Osborn et al. (17) did a meta-analysis focusing on the prevalence of post-TBI generalized anxiety disorder (GAD). The results showed that approximately $11 \%$ of the patients were diagnosed with GAD after TBI, ranging from 2 to $28 \%$. Taking into consideration the type of instrument used, the diagnostic scale ICD-10 was related to a lower prevalence rate $(2 \%)$ of GAD after TBI, whereas the DSM-III-R was related to a higher prevalence (19\%) of GAD (17). For interview schedules, SCAN showed a lower prevalence of GAD (2\%), while the Schedule for Affective Disorders and Schizophrenia (SADS) showed a higher prevalence (28\%) (17). The authors report differences in anxiety rates depending on the stages of TBI recovery. Thus, the timing of assessment may impact the number and severity of the symptoms, leading to bias in the results (158). The meta-analysis also 
TABLE 5 | Characteristics of the studies and the scales and inventories used to assess PTSD after TBI.

\begin{tabular}{|c|c|c|c|c|}
\hline Reference & Study design & Study population & $\begin{array}{l}\text { Number of analyzed } \\
\text { studies/type of } \\
\text { studies }\end{array}$ & Instruments \\
\hline Betthauser et al. (108) & Systematic review & Military veterans with TBI & $(30 / 47)$ & $\begin{array}{l}\text { CAPS, DSM-IV, DSM-IV-TR, DTS NSI, PCL, PCL-C, } \\
\text { PCL-M, PC-PTSD, TSI }\end{array}$ \\
\hline Brady et al. (109) & Review & Veterans with PTSD, SUD, TBI & N/A & $\begin{array}{l}\text { CAPS, IES-R, MPSS-SR, M-PTSD, NWS-PTSD, } \\
\text { PCL-M, PSEI, SUD }\end{array}$ \\
\hline Capehart and Bass (19) & Review & Veterans with TBI and PTSD & N/A & PCL \\
\hline Carlson et al. (110) & Systematic review & TBI & 31 & $\begin{array}{l}\text { BSI, CAPS, CIDI, IES, IES-R, PCL-M, PCL, PTSD-I, } \\
\text { PDS, PSS, PSE, SCID }\end{array}$ \\
\hline Carlson et al. (111) & Systematic review & mTBI/PTSD & 34 & CAPS, IES, PDS \\
\hline Cooper et al. (31) & Systematic review & Military veterans with mTBI & $(10 / 19)$ & CAPS, MPAI-4, NSI, PCL, PCL-C, PCL-M, PHQ \\
\hline Daggett et al. (33) & Systematic review & Veterans/military with TBI & $(1 / 17)$ & PCL-C, SCID \\
\hline Garrelfs et al. (20) & Systematic review & $\mathrm{ABI}(\mathrm{TBI}=4)$ & $(1 / 7)$ & SCID-I \\
\hline Gill et al. (4) & Systematic review & TBI & 28 & CAPS, CIDI, IES, IES-R, SCID, PCL, PDS, PSS, PTSD-I \\
\hline Harvey et al. (112) & Review & $\mathrm{TBI}$ & $\mathrm{N} / \mathrm{A}$ & $\begin{array}{l}\text { CAPS, CIDI, DIS, DSM-IV, ICD-10, IES, PSS, PSE, Penn } \\
\text { Inventory, PTSD-I, SCID }\end{array}$ \\
\hline Hesdorffer et al. (37) & Systematic review & TBI & $(5 / N / A)$ & CIDI, CAPS \\
\hline Karr et al. (113) & Systematic review & Blast-related mTBI & 9 & CAPS \\
\hline Kennedy et al. (114) & Review & Military veterans with $\mathrm{mTBI}$ or PCS & N/A & DSM-III, DSM-III-R, DSM-IV, DSM-IV-TR \\
\hline Kim et al. (21) & Systematic review & $\mathrm{TBI}$ & $(16 / 66)$ & $\begin{array}{l}\text { CAPS, DSM-III-R, PSE, IES, CIDI, DSM-IV, PDS, } \\
\text { PTSD-I, SCID }\end{array}$ \\
\hline Matarazzo et al. (18) & Systematic review & Veterans/military with TBI & 3 & CAPS, PCL-S \\
\hline Moore et al. (99) & Review & mTBI & N/A & DSM-III-R, MCMI-III, MMPI \\
\hline O’Neil et al. (23) & Systematic review & Veterans/military with mTBI & $(17 / 31)$ & CAPS, PCL, PCL-C, PCL-M, PCL-S, SCID \\
\hline O’Neil et al. (22) & Systematic review & Veterans/military with mTBI & $(17 / 31)$ & CAPS, PCL, PCL-C, PCL-M, PCL-S, SCID \\
\hline McMillan et al. (115) & Review & $\mathrm{TBl}$ & N/A & DSM-III, DSM-III-R, DSM-IV, IES \\
\hline Rice and Sher (116) & Review & Veterans with TBI & $\mathrm{N} / \mathrm{A}$ & DSM, PCL, PHQ \\
\hline Rogers and Read (25) & Review & $\mathrm{TBI}$ & $(7 / N / A)$ & BEC, IES, HSCL, PTSD-I, SCL-90-R, SCID \\
\hline Soo and Tate (26) & Systematic review & $\mathrm{mTBI}$ & $(1 / 3)$ & CAPS, IES \\
\hline Steel et al. (45) & Systematic review & Traumatic injury & $\mathrm{N} / \mathrm{A}$ & $\begin{array}{l}\text { PTSD-I, CAPS, CIDI, SCID, SI-PTSD, DIS, PDS, IES-R, } \\
\text { PC-PTSD, PCL, HTQ, M-PTSD, Civilian-MSS, Purdue } \\
\text { PTSD, Penn Inventory, TSI }\end{array}$ \\
\hline Tanev et al. (117) & Systematic review & $\mathrm{TBI}$ & $\mathrm{N} / \mathrm{A}$ & ANAM, CAPS, DTS, NSI, PSS, PCL, SI-PTSD, TSI \\
\hline Trachtman (118) & Review & Veterans with TBI & N/A & $\begin{array}{l}\text { DSM-IV, Halstead-Reitan test, ICD-9, MMPI, PCL-M, } \\
\text { PDHA, PDHRA }\end{array}$ \\
\hline Wall (119) & Systematic review & $\begin{array}{l}\text { TBI in military and veteran } \\
\text { population }\end{array}$ & 20 & $\begin{array}{l}\text { ANAM, BSI, DSM-IV, ICD-9, ICD-10PCL, M-PTSD, NSI, } \\
\text { PCL-M, PCL-C, PDHA, PDHRA, PHQ }\end{array}$ \\
\hline
\end{tabular}

PTSD, posttraumatic stress disorder; mTBI, mild traumatic brain injury.

Scales: ASDI, Acute Stress Disorder Interview; CAPS, Clinician-Administered PTSD Scale; CIDI, Composite International Diagnostic Interview; CSQ, Coping Style Questionnaire; Civilian-MSS, Civilian Mississippi Scale; DTS, Davidson Trauma Scale; DIS, Diagnostic Interview Schedule; M-PTSD, Mississippi Scale for Combat-Related PTSD; IES, Impact of Events Scale; IES-R, Impact of Events Scale-Revised; HTQ, Harvard Trauma Questionnaire; PCL, PTSD Checklist; PCL-C, PTSD Checklist - Civilian version; PCL-M, PTSD Checklist - Military version; PCL-S, PTSD Checklist - Stressor specific; PC-PTSD, Primary Care PTSD Screen; PDS, Posttraumatic Diagnostic Scale; PSE, Present State Examination; PSS, Posttraumatic Stress Scale; PSS-I, PTSD Symptoms Scale-Interview; SI-PTSD, Structured Interview for PTSD; PTSD-I, PTSD interview; SCID, Structured Clinical Interview for DSM; TSI, Trauma Symptoms Inventory.

showed a non-significant increase in the number of anxiety cases in the first 5 years post-trauma (17).

Two anxiety scales are suggested by the CDE for TBI populations: the Kiddie-Schedule for Affective Disorders and Schizophrenia (K-SADS) and Neuropsychiatric Rating Schedule (NRS). Interestingly, these scales were not extensively reported in this review, and the NRS was not reported in any of them.

The authors also recommend assessing substance abuse as a comorbidity of psychiatric conditions, especially in anxiety disorders $(22,24)$. The CDE suggests some questionnaires for this purpose: the Substance Abuse Questions from the TBI Model Systems Database, Alcohol Use Disorders Identification Test: selfreported version (AUDIT), and Alcohol, Smoking, and Substance Use Involvement Screening Test (ASSIST).

\section{Posttraumatic Stress Disorder}

Posttraumatic stress disorder and GAD showed high prevalence after TBI and were both classified as anxiety disorders. In 2013, the DSM-5 classified PTSD as a trauma-stressor-related disorder, rather than an anxiety disorder. For this reason, we classified PTSD and anxiety disorders as separate psychiatric conditions.

In TBI patients, PTSD is usually related to a severe accident or injury, violent assault, domestic violence, war, or disaster (Criterion A - DSM-5). Prevalence rates of PTSD after TBI range from 3 to $59 \%(159,160)$, while $43.9 \%$ of soldiers who reported loss of consciousness post-TBI met the criteria for PTSD (161). Our findings support previous results in which the heterogeneous range of diagnoses is due to the differences in assessment methods and methodologies of the original studies (111). 
TABLE 6 | Scales and inventories used to assess posttraumatic stress disorder (PTSD) after TBI.

\begin{tabular}{|c|c|c|}
\hline Name of the scale & Abbreviation & Clinical utility \\
\hline Acute Stress Disorder Interview (120) & ADIS & Interview schedule \\
\hline Brief Symptoms Inventory (53) & BSI & Interview schedule \\
\hline Civilian Mississippi Scale (121) & Civilian-MSS & Interview schedule \\
\hline Clinician-Administered Posttraumatic Stress Disorder Scale (122) & CAPS & Interview Schedule \\
\hline Composite International Diagnostic Interview (58) & $\mathrm{CIDI}$ & Interview schedule \\
\hline Coping Style Questionnaire (123) & $\mathrm{CSQ}$ & Interview Schedule \\
\hline Diagnostic and Statistical Manual 3rd edition [DSM-III: $n$ (63)] & DSM-III & Diagnose \\
\hline Diagnostic and Statistical Manual 3rd edition-revised [DSM-III-R (100)] & DSM-III-R & Diagnose \\
\hline Diagnostic and Statistical Manual 4th edition [DSM-IV $(64,123)]$ & DSM-IV & Diagnose \\
\hline Diagnostic and Statistical Manual 4th edition text revision [DSM-IV-TR (124)] & DSM-IV-TR & Diagnose \\
\hline Diagnostic and Statistical Manual 5th edition [DSM-5 (125)] & DSM-5 & Diagnose \\
\hline Davidson Trauma Scale (126) & DTS & Interview schedule \\
\hline Diagnostic Interview Schedule (83) & DIS & Interview schedule \\
\hline Harvard Trauma Questionnaire (127) & HTQ & Interview schedule \\
\hline Hopkins Symptom Checklist (69) & HSCL & Interview schedule \\
\hline International Classification of Diseases 9th edition [International Classification of Diseases (ICD) (71) & ICD-9 & Diagnose \\
\hline International Classification of Diseases 10th edition (72) & ICD-10 & Diagnose \\
\hline Impact of Events Scale (101) & IES & Interview schedule \\
\hline Impact of Events Scale-Revised (128) & IES-R & Interview schedule \\
\hline Mayo-Portland Adaptability Inventory-4 $(74,129)$ & MPAI-4 & Interview schedule \\
\hline Mississippi Scale for Combat-Related Posttraumatic Stress Disorder (130) & M-PTSD & Interview schedule \\
\hline Modified Posttraumatic Stress Disorder Symptom Scale (131) & MPSS-SR & Interview schedule \\
\hline National Women's Study Posttraumatic Stress Disorder module (132) & NWS-PTSD & Interview schedule \\
\hline Patient Health Questionnaire (85) & $\mathrm{PHQ}$ & Interview schedule \\
\hline Penn Inventory for Posttraumatic Stress Disorder (133) & Penn inventory & Interview schedule \\
\hline Post-Deployment Health Assessment (134) & PDHA & Interview schedule \\
\hline Post-Deployment Health Reassessment (135) & PDHRA & Interview schedule \\
\hline Posttraumatic Diagnostic Scale (136) & PDS & Interview schedule \\
\hline Posttraumatic Stress Scale (137) & PSS & Interview schedule \\
\hline Potential Stressful Events Interview (138) & PSEl & Interview schedule \\
\hline Present State Examination (87) & PSE & Interview schedule \\
\hline Primary Care Posttraumatic Stress Disorder Screen (139) & PC-PTSD & Interview schedule \\
\hline Posttraumatic Stress Disorder Checklist $(140,141)$ & PCL & Interview schedule \\
\hline Posttraumatic Stress Disorder Checklist - Civilian version (142) & PCL-C & Interview schedule \\
\hline Posttraumatic Stress Disorder Checklist - Military version (143) & PCL-M & Interview schedule \\
\hline Posttraumatic Stress Disorder Checklist - Stressor specific (144) & PCL-S & Interview schedule \\
\hline Posttraumatic Stress Disorder Interview (145) & PTSD-I & Interview schedule \\
\hline Self-rating Posttraumatic Stress Disorder Inventory (146) & SIP & Interview schedule \\
\hline Posttraumatic Stress Disorder Inventory Revised (147) & Revised PTSD inventory & Interview schedule \\
\hline Posttraumatic Stress Disorder Symptoms Scale-Interview (137) & PSS-I & Interview schedule \\
\hline Purdue Posttraumatic Stress Disorder (148) & Purdue PTSD & Interview schedule \\
\hline Symptoms Checklist 90-Revised (106) & SCL-90-R & Interview schedule \\
\hline Structured Clinical Interview for DSM (105) & SCID & Diagnose \\
\hline Structured Interview for Posttraumatic Stress Disorder (149) & SI-PTSD & Interview schedule \\
\hline Trauma Symptoms Inventory (150) & TSI & Interview schedule \\
\hline
\end{tabular}

Depression, anxiety, and PTSD are usually comorbid conditions following TBI and may facilitate the persistence of its effects (162). Gill et al. (4) showed that psychological well-being is not predictive of the development of PTSD, but evidence suggests that individuals who have a history of psychological difficulties are at greater risk of developing PTSD after TBI.

Posttraumatic stress disorder is one of the most common mental health disorders affecting approximately $15 \%$ of veterans with no history of TBI. Nevertheless, the diagnosis rates of PTSD range from 33 to $65 \%$ in veterans with a history of TBI (163, 164). For this sample, the PCL is broadly used. The PCL-M and
CAPS are the interview schedules most commonly used to assess PTSD in veterans and are recommended by the CDE (15). CAPS is considered the "gold standard" instrument for diagnosing and measuring the severity of PTSD, and it has been used with a variety of traumatized populations, including TBI $(109,165)$. There are different versions available, including CAPS to assess past-week, past-month, and lifetime symptoms. The PCL is a 17-item self-reported measure of PTSD symptoms and requires less time to complete than CAPS, which consists of a 30-item self-reported questionnaire. PCL is highly correlated with CAPS $(r=0.93)$, and it has favorable diagnostic efficiency $(>0.70)$ and 
robust psychometric properties (165). For civilians with TBI, the PCL and CAPS are the most commonly used instruments to assess PTSD. However, the PCL-C and PTSD Checklist - Stressor specific (PCL-S) are preferable.

Overall, psychological variables, worsening general health, chronic pain, and somatic symptoms are associated with PTSD, especially in moderate to severe TBI. Comorbidities, such as PTSD and TBI, may unfavorably affect individuals more than suffering from any disorder alone (166). Some factors suggest how individuals with TBI might be more likely to develop PTSD. Somatic conditions and psychiatric disorders, such as PTSD, seem to perpetuate the illness condition in a loop (167). Those somatic conditions may present not only as risk factors but may also contribute to the persistence of other disorders, such as PTSD (4). This highlights the importance of therapy and rehabilitation for PTSD after TBI.

\section{Brain Function and Mental Health Post-TBI}

Symptoms of anxiety, depression, and irritability often occur after TBI and affect mood centers, including the hippocampus, amygdala, and prefrontal regions of the brain (168). Psychological factors are potential contributors to poor recovery after mTBI (8). Since TBI etiologies are diverse, understanding the role of the neurobiological basis for behavioral dysfunctions can be complex. The neuroanatomical location of the head injury can play a role in the development of depression $(45,169)$. However, only a small portion of patients may sustain damage to the particular location and with a severity level necessary to produce a psychiatric syndrome while preserving adequate cognitive function; thus, a biological gradient can be very difficult to detect (25). Premorbid factors associated with psychiatric disorders are inconclusive. Family history of psychiatric disorders seems to be a predictor of depression (169) or PTSD in individuals who have experienced TBI (45). In addition, females have a higher risk of developing acute PTSD after motor vehicle accidents (170). However, some authors did not find this association in premorbid psychiatric illness with the development of PTSD (171).

Acquired brain lesions, especially those involving the prefrontal cortex may have a prominent role in developing and maintaining executive functions. These functions encompass a set of skills that allow for people's adjustment and adaptation in the face of new situations and daily operation. Therefore, changes in executive functions are among the most common consequences resulting from TBI $(172,173)$. Depressive symptoms can also affect cognitive processes, inhibiting a patient's ability to return to daily activities over the short-term. Prefrontal cortex disruption may result in impulsive behaviors and a higher risk of substance use disorders (174). Thus, there is evidence that TBI may increase the risk of drug or alcohol abuse in persons without a history of substance abuse before the injury, especially if the damage involves the orbitofrontal cortex (175). In our review, only short reports attempted to find associations between mental health and substance abuse. Hesdorffer et al. (37) reported that changes in drug and alcohol use usually preceded the occurrence of TBI, increasing the risk of head injury. One important point is that many of the studies analyzed in the reviews used current alcohol or substance abuse as exclusion criteria, possibly camouflaging their frequency of occurrence.

\section{Perspectives and Future Research}

Considering the high incidence of TBI in the elderly (140-200 per 100,000 per year) and the relatively high prevalence of depression following TBI, it is reasonable to address the question of depression in the elderly following TBI (38). There is a gap in the literature addressing the evaluation and monitoring of elderly TBI patients.

One limitation related to veterans and military populations is that most of the analyzed data are from medical registries and clinical databases. It is necessary to have larger cohort studies, and it is also necessary to use standard methodology for the assessment of veterans and military populations.

Even in systematic reviews and meta-analyses, attrition bias may occur, as some studies showed that individuals who did not complete studies generally had more severe TBI (176-179) or, conversely, had less severe TBI (180). In addition, some studies did not control for confounding variables, such as the severity of trauma or the period post-TBI, reducing the generalizability of some results.

Many studies utilized self-report or semi-structured interviews for diagnosis. These results can lead to bias in the reported frequency of depressive disorder in this population. Self-report scales may not be reliable as patients with TBI may be unaware of their disabilities, and lack of awareness may lead to an underdiagnosis of psychiatric disorders (25). Another important variable relates to the recruitment of study participants. Outpatient and inpatient populations tend to vary in the intensity of their symptoms, particularly in the acute stage of trauma, when patients are often confused and disoriented. Many reviews analyzed mixed samples of trauma severity and different recruitment settings (17). Moreover, the majority of the instruments require that patients report their symptoms over the previous 2 weeks, and patients may have difficulty answering such questions or giving reliable responses, particularly in the acute stages of trauma.

Another important issue is the analysis of premorbid psychiatric conditions, a factor that may bias results. Finally, as we are reporting on specific information from selected reviews in the field, there is the risk of publication bias.

\section{CONCLUSION}

There is significant variability in the types of assessments used in the evaluation of psychiatric disorders after TBI, and consequently, there is also variability in the reported prevalence of such disorders. We analyzed meta-analyses and systematic reviews focusing on the most prevalent psychiatric conditions, and we observed a heterogeneous pattern related to their assessment and diagnosis in TBI populations. Depression after TBI is a well-established condition with homogeneous studies. Anxiety and PTSD disorders have been studied in a heterogeneous way, usually comorbid with other psychiatric disorders. The variability of clinical findings raises the importance of the instruments used 
to assess these patients. Finally, some scales and inventories designed for the general community may not be appropriate for patients with TBI (152).

\section{AUTHOR CONTRIBUTIONS}

$\mathrm{AZ}$ - had the idea of the review, organized the search method, and wrote the manuscript. JV - helped to write the manuscript and did the tables. FF - reviewed the manuscript and helped the

\section{REFERENCES}

1. Faul M, Xu L, Wald MM, Coronado V, Dellinger AM. Traumatic brain injury in the United States: national estimates of prevalence and incidence, 20022006. Injury Prev (2010) 16:A268-268. doi:10.1136/ip.2010.029951

2. Nitrini R, Bacheschi LA. A Neurologia que Todo o Medico Deve Saber. 2 ed. São Paulo: Editora Atheneu (2003) 503 p.

3. De Souza CAC. Neuropsiquiatria dos traumatismos craniencefálicos. Revinter: Rio de Janeiro (2003).

4. Gill J, LeeH, BarrT, Baxter T, Heinzelmann M, RakH, et al.Lower health related quality of life in US military personnel is associated with service-related disorders and inflammation. Psychiatry Res (2014) 216(1):116-22. doi:10.1016/j. psychres.2014.01.046

5. Menon DK, Schwab K, Wright DW, Maas AI. Position statement: definition of traumatic brain injury. Arch Phys Med Rehabil (2010) 91(11):1637-40. doi:10.1016/j.apmr.2010.05.017

6. Nitrini R, Bacheschi LA. A neurologia que todo médico deve saber. Maltese (1991).

7. Arciniegas DB, Wortzel HS. Emotional and behavioral dyscontrol after traumatic brain injury. Psychiatr Clin North America (2014) 37(1):31-53. doi:10.1016/j.psc.2013.12.001

8. Ponsford J, Cameron P, Fitzgerald M, Grant M, Mikocka-Walus A. Long-term outcomes after uncomplicated mild traumatic brain injury: a comparison with trauma controls. JNeurotrauma (2011) 28(6):937-46. doi:10.1089/ neu.2010.1516

9. Ponsford JL, Downing MG, Olver J, Ponsford M, Acher R, Carty M, et al. Longitudinal follow-up of patients with traumatic brain injury: outcome at two, five, and ten years post-injury. J Neurotr (2014) 31(1):64-77. doi:10.1089/ neu.2013.2997

10. Riggio S. Traumatic brain injury and its neurobehavioral sequelae. Psychiatric Clin North America (2010) 33(4):807-19. doi:10.1016/j.psc.2010.08.004

11. Inglese M, Makani S, Johnson G, Cohen BA, Silver JA, Gonen O, et al. Diffuse axonal injury in mild traumatic brain injury: a diffusion tensor imaging study. J Neurosurg (2005) 103(2):298-303. doi:10.3171/jns.2005.103.2.0298

12. Silver JM, McAllister TW, Arciniegas DB. Depression and cognitive complaints following mild traumatic brain injury. Am J Psychiatry (2009) 166(6):653-61. doi:10.1176/appi.ajp.2009.08111676

13. Jorge RE, Starkstein SE. Pathophysiologic aspects of major depression following traumatic brain injury. J Head Trauma Rehabil (2005) 20(6):475-87. doi:10.1097/00001199-200511000-00001

14. Gordon WA, Zafonte R, Cicerone K, Cantor J, Brown M, Lombard L, et al. Traumatic brain injury rehabilitation - state of the science. Am J Phys Med Rehabil (2006) 85(4):343-82. doi:10.1097/01.phm.0000202106.01654.61

15. Maas AI, Harrison-Felix CL, Menon D, Adelson PD, Balkin T, Bullock R, et al. Common data elements for traumatic brain injury: recommendations from the interagency working group on demographics and clinical assessment. Arch Phys Med Rehabil (2010) 91(11):1641-9. doi:10.1016/j. apmr.2010.07.232

16. Fulton JJ, Calhoun PS, Wagner HR, Schry AR, Hair LP, Feeling N, et al. The prevalence of posttraumatic stress disorder in Operation Enduring Freedom/ Operation Iraqi Freedom (OEF/OIF) Veterans: a meta-analysis. J Anxiety Disord (2015) 31:98-107. doi:10.1016/j.janxdis.2015.02.003

17. Osborn AJ, Mathias JL, Fairweather-Schmidt AK. Depression following adult, non-penetrating traumatic brain injury: a meta-analysis examining elaboration of the manuscript. PR - helped to write the manuscript. CB - helped on the tables review. ML - helped to review the manuscript. WP - reviewed the manuscript.

\section{FUNDING}

We thank the National Counsel of Technological and Scientific Development, process number: 201809/2014-1 for the $\mathrm{PhD}$ scholarship.

methodological variables and sample characteristics. Neurosci Biobehav Rev (2014) 47:1-15. doi:10.1016/j.neubiorev.2014.07.007

18. Matarazzo BB, Wortzel HS, Holliman BAD, Brenner LA. Evidence-based intervention strategies for veterans and military personnel with traumatic brain injury and co-morbid mental health conditions: a systematic review. Brain Impairment (2013) 14(1):42-50. doi:10.1017/BrImp.2013.4

19. Capehart B, Bass D. Review: managing posttraumatic stress disorder in combat veterans with comorbid traumatic brain injury. J Rehabil Res Dev (2012) 49(5):789-812. doi:10.1682/jrrd.2011.10.0185

20. Garrelfs SF, Donker-Cools B, Wind H, Frings-Dresen MHW. Return-to- work in patients with acquired brain injury and psychiatric disorders as a comorbidity: a systematic review. Brain Injury (2015) 29(5):550-7. doi:10.3109/02 699052.2014.995227

21. Kim E, Lauterbach EC, Reeve A, Arciniegas DB, Coburn KL, Mendez MF, et al. Neuropsychiatric complications of traumatic brain injury: a critical review of the literature (A report by the ANPA Committee on Research). J Neuropsychiatr Clin Neurosci (2007) 19(2):106-27. doi:10.1176/appi. neuropsych.19.2.106

22. O’Neil M, Carlson K, Storzbach D, Brenner L, Freeman M, Quinones A, et al. A systematic review of consequences of mild traumatic brain injury in veterans and members of the military. Arch Clin Neuropsychol (2013) 28(6):606-606.

23. O'Neil ME, Carlson KF, Storzbach D, Brenner LA, Freeman M, Quinones AR, et al. Factors associated with mild traumatic brain injury in veterans and military personnel: a systematic review. JInt Neuropsychol Soc (2014) 20(3):249-61. doi:10.1017/s135561771300146x

24. Panayiotou A, Jackson M, Crowe SF. A meta-analytic review of the emotional symptoms associated with mild traumatic brain injury. J Clin Exp Neuropsychol (2010) 32(5):463-73. doi:10.1080/13803390903164371

25. Rogers JM, Read CA. Psychiatric comorbidity following traumatic brain injury. Brain Inj (2007) 21(13-14):1321-33. doi:10.1080/02699050701765700

26. Soo C, Tate R. Psychological treatment for anxiety in people with traumatic brain injury. Cochrane Database Syst Rev (2007) 18(3):CD005239. doi:10.1002/14651858.CD005239.pub2

27. Warden DL, Gordon B, McAllister TW, Silver JM, Barth JT, Bruns J, et al. Guidelines for the pharmacologic treatment of neurobehavioral sequelae of traumatic brain injury. J Neurotrauma (2006) 23(10):1468-501. doi:10.1089/ neu.2006.23.1468

28. Liberati A, Altman DG, Tetzlaff J, Mulrow C, Gotzsche PC, Ioannidis JPA, et al. The PRISMA statement for reporting systematic reviews and meta-analyses of studies that evaluate health care interventions: explanation and elaboration. Ann Intern Med (2009) 151(4):W65-94. doi:10.7326/0003-4819-151-4-200908180-00136

29. Adamson BC, Ensari I, Motl RW. Effect of exercise on depressive symptoms in adults with neurologic disorders: a systematic review and meta-analysis. Arch Phys Med Rehabil (2015) 96(7):1329-38. doi:10.1016/j.apmr.2015.01.005

30. Barker-Collo S, Starkey N, Theadom A. Treatment for depression following mild traumatic brain injury in adults: a meta-analysis. Brain Injury (2013) 27(10):1124-33. doi:10.3109/02699052.2013.801513

31. Cooper DB, Bunner AE, Kennedy JE, Balldin V, Tate DF, Eapen BC, et al. Treatment of persistent post-concussive symptoms after mild traumatic brain injury: a systematic review of cognitive rehabilitation and behavioral health interventions in military service members and veterans. Brain Imaging Behav (2015) 9(3):403-20. doi:10.1007/s11682-015-9440-2 
32. Crisp R. Depression and occupational disability in five diagnostic groups: a review of recent research. Disabil Rehabil (2007) 29(4):267-79. doi:10.1080/09638280600835267

33. Daggett V, Bakas T, Habermann B. A review of health-related quality of life in adult traumatic brain injury survivors in the context of combat veterans. J Neurosci Nursing (2009) 41(2):59-71. doi:10.1097/JNN.0b013e31819a7133

34. Fann JR, Hart T, Schomer KG. Treatment for depression after traumatic brain injury: a systematic review. J Neurotrauma (2009) 26(12):2383-402. doi:10.1089/neu.2009.1091

35. Fleminger S, Oliver DL, Williams WH, Evans J. The neuropsychiatry of depression after brain injury. Neuropsychol Rehabil (2003) 13(1-2):65-87. doi:10.1080/09602010244000354

36. Halbauer JD, Ashford JW, Zeitzer JM, Adamson MM, Lew HL, Yesavage JA. Neuropsychiatric diagnosis and management of chronic sequelae of war-related mild to moderate traumatic brain injury. J Rehabil Res Dev (2009) 46(6):757-95. doi:10.1682/jrrd.2008.08.0119

37. Hesdorffer DC, Rauch SL, Tamminga CA. Long-term psychiatric outcomes following traumatic brain injury: a review of the literature. J Head Trauma Rehabil (2009) 24(6):452-9. doi:10.1097/HTR.0b013e3181c133fd

38. Menzel JC. Depression in the elderly after traumatic brain injury: a systematic review. Brain Inj (2008) 22(5):375-80. doi:10.1080/02699050802001492

39. Nowrangi MA, Kortte KB, Rao VA. A perspectives approach to suicide after traumatic brain injury: case and review. Psychosomatics (2014) 55(5):430-7. doi:10.1016/j.psym.2013.11.006

40. Oyesanya TO, Ward EC. Mental health in women with traumatic brain injury: a systematic review on depression and hope. Health Care Women Int (2016) 37(1):45-74. doi:10.1080/07399332.2015.1005307

41. Rosenthal M, Christensen BK, Ross TP. Depression following traumatic brain injury. Arch Phys Med Rehabil (1998) 79(1):90-103. doi:10.1016/ S0003-9993(98)90215-5

42. Sherer M, Davis LC, Sander AM, Caroselli JS, Clark AN, Pastorek NJ. Prognostic importance of self-reported traits/problems/strengths and environmental barriers/facilitators for predicting participation outcomes in persons with traumatic brain injury: a systematic review. Arch Phys Med Rehabil (2014) 95(6):1162-73. doi:10.1016/j.apmr.2014.02.006

43. Simpson G, Tate R. Suicidality in people surviving a traumatic brain injury: prevalence, risk factors and implications for clinical management. Brain Inj (2007) 21(13-14):1335-51. doi:10.1080/02699050701785542

44. Stalder-Luthy F, Messerli-Burgy N, Hofer H, Frischknecht E, Znoj H, Barth J. Effect of psychological interventions on depressive symptoms in long-term rehabilitation after an acquired brain injury: a systematic review and meta-analysis. Arch Phys Med Rehabil (2013) 94(7):1386-97. doi:10.1016/j. apmr.2013.02.013

45. Steel JL, Dunlavy AC, Stillman J, Pape HC. Measuring depression and PTSD after trauma: common scales and checklists. Injury (2011) 42(3):288-300. doi:10.1016/j.injury.2010.11.045

46. Vahle VJ, Andresen EM, Hagglund KJ. Depression measures in outcomes research. Arch Phys Med Rehabil (2000) 81(12):S53-62. doi:10.1053/ apmr.2000.20625

47. van Velzen JM, van Bennekom CAM, Edelaar MJA, Sluiter JK, Frings-Dresen MHW.Prognosticfactors of return to workafteracquiredbraininjury:asystematic review. Brain Inj (2009) 23(5):385-95. doi:10.1080/02699050902838165

48. Beck AT, Erbaugh J, Ward CH, Mock J, Mendelsohn M. An inventory for measuring depression. Arch Gen Psychiatry (1961) 4(6):561-71. doi:10.1001/ archpsyc.1961.01710120031004

49. Beck AT, Steer RA, Ball R, Ranieri WF. Comparison of Beck depression inventories-IA and -II in psychiatric outpatients. JPers Assess (1996) 67(3):588-97. doi:10.1207/s15327752jpa6703_13

50. Beck AT, Steer RA. Manual for the Beck Hopelessness Scale. San Antonio, TX: Psychological Corp (1988).

51. U.S. Department of Health \& Human Services, Center for Disease Control \& Prevention. Behavioral Risk Factor Surveillance System (BRFSS). Atlanta: Behavioral Risk Factor Surveillance System (BRFSS) (1984).

52. Overall JE, Gorham DR. The brief psychiatric rating-scale. Psychol Rep (1962) 10(3):799-812. doi:10.2466/pr0.1962.10.3.799

53. Derogatis LR. BSI Brief Symptom Inventory: Administration, Scoring, and Procedure Manual. 4 ed. Minneapolis, MN: National Computer Systems (1993).
54. Derogatis LR. Brief Symptom Inventory (BSI) 18: Administration, Scoring, and Procedures Manual. Minneapolis, MN: NCS Pearson (2001).

55. Devins GM, Orme CM, Costello CG, Binik YM, Frizzell B, Stam HJ, et al. Measuring depressive symptoms in illness populations: psychometric properties of the Center for Epidemiologic Studies Depression (CES-D) Scale. Psychol Health (1988) 2(2):139-56. doi:10.1080/08870448808400349

56. Krug SE. Development of a validity scale for the clinical analysis questionnaire. Multivar Exp Clin Res (1979) 4(4):125-31.

57. Botega NJ, Zomignani MA, Garcia C Jr, Bio MR, Pereira WAB. Psychiatric morbidity in the general hospital: the use of the revised clinical interview schedule (CIS-R). Revista ABP-APAL (1994) 16(2):57-62.

58. Robins LN, Wing J, Wittchen HU, Helzer JE, Babor TF, Burke J, et al. The composite international diagnostic interview - an epidemiologic instrument suitable for use in conjunction with different diagnostic systems and in different cultures. Arch Gen Psychiatry (1988) 45(12):1069-77. doi:10.1001/ archpsyc.1988.01800360017003

59. Asberg M, Montgomery SA, Perris C, Schalling D, Sedvall G. Comprehensive psychopathological rating-scale. Acta Psychiatr Scand (1978) 271:5-27. doi:1 0.1111/j.1600-0447.1978.tb02357.x

60. Lubin B. Adjective checklists for measurement of depression. Arch Gen Psychiatry (1965) 12(1):57-62. doi:10.1001/archpsyc.1965.01720310059007

61. Lovibond SH, Lovibond PF. Manual for the Depression Anxiety Stress Scale. Sydney: Psychology Foundation (1995).

62. Turner-Stokes L, Kalmus M, Hirani D, Clegg F. The Depression Intensity Scale Circles (DISCS): a first evaluation of a simple assessment tool for depression in the context of brain injury. J Neurol Neurosurg Psychiatry (2005) 76(9):1273-8. doi:10.1136/jnnp.2004.050096

63. American Psychiatric Association. DSM-III: Diagnostic and Statistical Manual of Mental Disorders. 3rd ed. Washington, DC: American Psychiatric Association (1980).

64. American Psychiatric Association. DSM-IV: Diagnostic and Statistical Manual of Mental Disorders. 4th ed. Washington, DC: American Psychiatric Association (1994).

65. Regier DA, Myers JK, Kramer M, Robins LN, Blazer DG, Hough RL, et al. The NIMH epidemiologic catchment-area program - historical context, major objectives, and study population characteristics. Arch Gen Psychiatry (1984) 41(10):934-41. doi:10.1001/archpsyc.1984.01790210016003

66. Goldberg DP, Hillier VF. Scaled version of the general health questionnaire. Psychol Med (1979) 9(1):139-45. doi:10.1017/S0033291700021644

67. Yesavage JA, Brink TL, Rose TL, Lum O, Huang V, Adey M, et al. Development and validation of a geriatric depression screening scale a preliminary report. J Psychiatr Res (1982) 17(1):37-50. doi:10.1016/0022-3956(82)90033-4

68. Hamilton M. A rating scale for depression. J Neurol Neurosurg Psychiatry (1960) 23:56-62.doi:10.1136/jnnp.23.1.56

69. Derogati LR, Lipman RS, Rickels K, Uhlenhut E, Covi L. Hopkins symptom checklist (HSCL) - self-report symptom inventory. Behav Sci (1974) 19(1):1-15. doi:10.1002/bs.3830190102

70. Zigmond AS, Snaith RP. The hospital anxiety and depression scale. Acta Psychiatr Scand (1983) 67(6):361-70. doi:10.1111/j.1600-0447.1983. tb09716.x

71. World Health Organization. ICD-9 International Statistical Classification of Diseases and Related Health Problems. 9th ed. Geneva: WHO ICD-99 (1978).

72. World Health Organization. ICD-10 International Statistical Classification of Diseases and Related Health Problems. 10th ed. Geneva: WHO ICD-10 (1992). Illus, IV+1243P-IV+1243P.

73. Snaith RP, Bridge GWK, Hamilton M. Leeds scales for self-assessment of anxiety and depression. Br J Psychiatry (1976) 128:156-65. doi:10.1192/ bjp.128.2.156

74. Malec JF. The Mayo-Portland participation index: a brief and psychometrically sound measure of brain injury outcome. Arch Phys Med Rehabil (2004) 85(12):1989-96. doi:10.1016/j.apmr.2004.01.032

75. Overholser JC, Schubert DSP, Foliart R, Frost F. Assessment of emotional distress following a spinal-cord injury. Rehabil Psychol (1993) 38(3):187-98. doi:10.1037//0090-5550.38.3.187

76. Millon T. Millon Clinical Multiaxial Inventory. 3rd ed. Minneapolis: National Computer System (1983).

77. Lecrubier Y, Sheehan DV, Weiller E, Amorim P, Bonora I, Sheehan KH, et al. The Mini International Neuropsychiatric Interview (MINI). A short 
diagnostic structured interview: reliability and validity according to the CIDI. Eur Psychiatry (1997) 12(5):224-31. doi:10.1016/s0924-9338(97)83296-8

78. Butcher JN, Dahlstrom WG, Graham JR, Tellegen A, Kaemmer B. The Minnesota Multiphasic Personality Inventory-2 (MMPI-2): Manual for Administration and Scoring. Minneapolis, MN: University of Minnesota Press (1989).

79. Schiele BC, Baker AB, Hathaway SR. The Minnesota multiphasic personality inventory. Lancet (1943) 63:292-7.

80. Montgomery SA, Asberg M. New depression scale designed to be sensitive to change. Br J Psychiatry (1979) 134:382-9. doi:10.1192/bjp.134.4.382

81. Costa PT Jr, McCrae RR. NEO PI-R Professional Manual. FL: Psychological Assessment Resources, Inc (1992).

82. Kreutzer JS, Marwitz JH, Seel R, Serio CD. Validation of a neurobehavioral functioning inventory for adults with traumatic brain injury. Arch Phys Med Rehabil (1996) 77(2):116-24. doi:10.1016/s0003-9993(96)90155-0

83. Helzer JE, Robins LN, Ratcliff KS. Validity of the NIMH diagnostic interview schedule (DIS) - (a psychiatric diagnostic interview for use by non-physicians in survey-research). J Psychiatr Res (1981) 16(2):133-4. doi:10.1016/0022-3956(81)90037-6

84. Vonkorff MR, Anthony JC. The NIMH diagnostic interview schedule modified to record current mental status. J Affect Disord (1982) 4(4):365-71. doi:10.1016/0165-0327(82)90032-5

85. Kroenke K, Spitzer RL, Williams JBW. The PHQ-9 - validity of a brief depression severity measure. JGen Intern Med (2001) 16(9):606-13. doi:10.1046/j.1525-1497.2001.016009606.x

86. Strack S, Lorr M. Item factor structure of the personality adjective check list. J Pers Assess (1990) 55(1-2):86-94. doi:10.1207/s15327752jpa5501\&2_9

87. Wing JK, Cooper JE, Sartorius N. Prsesent State Examination (PSE). Measurement and Classification of Psychiatric Symptoms: An Instruction Manual for the PSE and Catego Program. London: Cambridge University Press (1974).

88. McNair DM, Lorr M, Droppleman LF. Profile of Mood States. San Diego: Educational and Industrial Testing Service (1971).

89. Endicott J, Spitzer RL. Diagnostic interview - schedule for affective-disorders and schizophrenia. Arch Gen Psychiatry (1978) 35(7):837-44. doi:10.1001/ archpsyc.1978.01770310043002

90. Wing J. SCAN - schedules for clinical-assessment in neuropsychiatry. Psychiatry (1990) 900:85-90.

91. Spielberger CD, Vagg PR, Barker LR, Donham GW, Westberry LG. The Factor Structure of the State-Trait Anxiety Inventory. Stress and Anxiety. (Vol. 7). Washington, DC: Hemisphere (1980).

92. First MB, Spitzer RL, Gibbon M, Williams JBW. Structured Clinical Interview for DSM-IV-TR Axis I Disorder. New York: Biometrics Research, New York State Psychiatric Institute (2002).

93. Derogatis LR. Administration, Scoring and Procedure Manual-I for the $R$ (Revised) Version. Baltimore: Johns Hopkins University School of Medicine (1977).

94. Ritsner M, Rabinowitz J, Slyuzberg M. The Talbieh brief distress inventory - a brief instrument to measure psychological distress among immigrants. Compr Psychiatry (1995) 36(6):448-53. doi:10.1016/s0010-440x(95)90253-8

95. Aitken RCB. Measurement of feelings using visual analogue scales. Proc Royal Soc Med (1969) 62(10):989-93.

96. Beaumont G, Seldrup J, Murphy JE, McMillin M, Gringras M. Comparison between doctors and patients assessments of anxiety and depression using a series of visual analog scales. J Pharmacol (1974) 5:6-6.

97. Coughlan AK. The Wimbledon Self-Reports Scale: emotional and mood appraisal. Clin Rehabil (1988) 2:207-13. doi:10.1177/026921558800200305

98. Zung WWK. A self-rating depression scale. Arch Gen Psychiatry (1965) 12:63-70. doi:10.1001/archpsyc.1965.01720310065008

99. Moore EL, Terryberry-Spohr L, Hope DA. Mild traumatic brain injury and anxiety sequelae: a review of the literature. Brain Inj (2006) 20(2):117-32. doi:10.1080/02699050500443558

100. American Psychiatric Association. DSM-III: Diagnostic and Statistical Manual of Mental Disorders. 3rd Revised ed. American Psychiatric Association: Washington, DC (1987).

101. Horowitz M, Wilner N, Alvarez W. Impact of event scale - measure of subjective stress. Psychosom Med (1979) 41(3):209-18. doi:10.1097/00006842-197905000-00004
102. The World Health Organization. The ICD-10 classification of mental and behavioural disorders - diagnostic criteria for research. Geneva: WHO (1993).

103. Bedford A. Manual of the Leeds scales for the self-assessment of anxiety and depression - Snaith, RP, Bridge, GWK, Hamilton, M. Br J Soc Clin Psychol (1979) 18:445-5.

104. Levin HS, Goldstein FC, MacKenzie EJ. Depression as a secondary condition following mild and moderate traumatic brain injury. Clin Neuropsychiatry (1997) 2(3):207-15.

105. First MB, Spitz RL, Gibbon M, Williams JBW. Structured Clinical Interview for DSM-IV Axis I Disorders - Patient Edition (SCID I/P, Version 2.0). New York, NY: Biometrics Research Department (1995).

106. Derogatis LR, Savitz KL. The SCL-90-R and brief symptom inventory (BSI) in primary care. Handbook of Psychological Assessment in Primary Care Settings. Mahwah: Lawrence Erlbaum Assoc Publ Ed. (2000). p. 297-334.

107. Goodman WK, Price LH, Rasmussen SA, Mazure C, Delgado P, Heninger GR, et al. The yale-brown obsessive compulsive scale 2. Validity. Arch Gen Psychiatry (1989) 46(11):1012-6.

108. Betthauser LM, Bahraini N, Krengel MH, Brenner LA. Self-report measures to identify post traumatic stress disorder and/or mild traumatic brain injury and associated symptoms in military veterans of operation enduring freedom (OEF)/Operation Iraqi Freedom (OIF). Neuropsychol Rev (2012) 22(1):35-53. doi:10.1007/s11065-012-9191-4

109. Brady KT, Tuerk P, Back SE, Saladin ME, Waldrop AE, Myrick H. Combat posttraumatic stress disorder, substance use disorders, and traumatic brain injury. J Addict Med (2009) 3(4):179-88. doi:10.1097/ADM.0b013e3181aa244f

110. Carlson K, Kehle S, Meis L, Greer N, MacDonald R, Rutks I, et al. The Assessment and Treatment of Individuals with History of Traumatic Brain Injury and Post-Traumatic Stress Disorder: A Systematic Review of the Evidence [Internet]. Washington, DC: Department of Veterans Affairs (US) (2009).

111. Carlson KF, Kehle SM, Meis LA, Greer N, MacDonald R, Rutks I, et al. Prevalence, assessment, and treatment of mild traumatic brain injury and posttraumatic stress disorder: a systematic review of the evidence. J Head Trauma Rehabil (2011) 26(2):103-15. doi:10.1097/HTR.0b013e3181e50ef1

112. Harvey AG, Brewin CR, Jones C, Kopelman MD. Coexistence of posttraumatic stress disorder and traumatic brain injury: towards a resolution of the paradox. J Int Neuropsychol Soc (2003) 9(4):663-76. doi:10.1017/ S1355617703940069

113. Karr JE, Areshenkoff CN, Duggan EC, Garcia-Barrera MA. Blastrelated mild traumatic brain injury: a Bayesian random-effects meta-analysis on the cognitive outcomes of concussion among military personnel. Neuropsychol Rev (2014) 24(4):428-44. doi:10.1007/s11065-0149271-8

114. Kennedy JE, Jaffee MS, Leskin GA, Stokes JW, Leal FO, Fitzpatrick PJ. Posttraumatic stress disorder and posttraumatic stress disorder-like symptoms and mild traumatic brain injury.J Rehabil Res Dev (2007) 44(7):895-919. doi:10.1682/JRRD.2006.12.0166

115. McMillan TM, Williams WH, Bryant R. Post-traumatic stress disorder and traumatic brain injury: a review of causal mechanisms, assessment, and treatment. Neuropsychol Rehabil (2003) 13(1-2):149-64. doi:10.1080/09602010244000453

116. Rice TR, Sher L. Suicidal behavior in war veterans. Expert Rev Neurother (2012) 12(5):611-24. doi:10.1586/ern.12.31

117. Tanev KS, Pentel KZ, Kredlow MA, Charney ME. PTSD and TBI co-morbidity: scope, clinical presentation and treatment options. Brain Inj (2014) 28(3):261-70. doi:10.3109/02699052.2013.873821

118. Trachtman JN. Post-traumatic stress disorder and vision. Optometry (2010) 81(5):240-52. doi:10.1016/j.optm.2009.07.017

119. Wall PLH. Posttraumatic stress disorder and traumatic brain injury in current military populations: a critical analysis. J Am Psychiatr Nurses Assoc (2012) 18(5):278-98. doi:10.1177/1078390312460578

120. Dinardo PA, Obrien GT, Barlow DH, Waddell MT, Blanchard EB. Reliability of DSM-III anxiety disorder categories using a new structured interview. Arch Gen Psychiatry (1983) 40(10):1070-4. doi:10.1001/ archpsyc.1983.01790090032005

121. Vreven DL, Gudanowski DM, King LA, King DW. The civilian version of the Mississippi PTSD Scale - a psychometric evaluation. J Trauma Stress (1995) 8(1):91-109. doi:10.1002/jts.2490080107 
122. Blake DD, Weathers FW, Nagy LM, Kaloupek DG, Gusman FD, Charney DS, et al. The development of a clinician-administered PTSD scale. J Trauma Stress (1995) 8(1):75-90. doi:10.1002/jts.2490 080106

123. Rosenstiel AK, Keefe FJ. The use of coping strategies in chronic low-back-pain patients - relationship to patient characteristics and current adjustment. Pain (1983) 17(1):33-44. doi:10.1016/0304-3959(83)90125-2

124. American Psychiatric Association. DSM-IV-TR: Diagnostic and Statistical Manual of Mental Disorders. 4th ed. American Psychiatric Association: Washington, DC(2000).

125. American Psychiatric Association. DSM-5: Diagnostic and Statistical Manual of Mental Disorders. 5th ed. Arlington, VA: American Psychiatric Publishing (2013).

126. Davidson JRT, Book SW, Colket JT, Tupler LA, Roth S, David D, et al. Assessment of a new self-rating scale for posttraumatic stress disorder. Psychol Med (1997) 27(1):153-60. doi:10.1017/S0033291796004229

127. Mollica RF, Caspiyavin Y, Bollini P, Truong T, Tor S, Lavelle J. The Harvard Trauma Questionnaire - validating a cross-cultural instrument for measuring torture, trauma, and posttraumatic-stress-disorder in Indo-Chinese refugees. JNerv Ment Dis (1992) 180(2):111-6. doi:10.1097/00005053-199202000-00008

128. Weiss DS, Marmar CR. The impact of event scale-revised. Assess Psychol Trauma PTSD (1997):399-411.

129. Malec JF, Moessner AM, Kragness M, Lezak MD. Refining a measure of brain injury sequelae to predict postacute rehabilitation outcome: rating scale analysis of the Mayo-Portland Adaptability Inventory. J Head Trauma Rehabil (2000) 15:670-82.

130. Keane TM, Caddell JM, Taylor KL. Mississippi Scale for combat-related posttraumatic stress disorder -3 studies in reliability and validity. J Consult Clin Psychol (1988) 56(1):85-90. doi:10.1037/0022-006X.56.1.85

131. Falsetti SA, Resnick HS, Resick PA, Kilpatrick D. The modified PTSD symptom scale: a brief self-report measure of posttraumatic stress disorder. Behav Ther (1993) 16:161-2.

132. Kilpatrick DG, Resnick HS, Saunders BE, Best CL. The National Woman's Study PTSD Module. Charleston, SC: Medical University of South Carolina (1989).

133. Hammarberg M. Penn Inventory for posttraumatic stress disorder: psychometric properties. Psychol Assess (1992) 4:67-76. doi:10.1037/1040-3590. 4.1.67

134. Population Health Support Division, Air Force Modernization Directorates, \& Office of the USAF Surgeon General. Post-Deployment Health Reassessment: Application User's Guide. San Antonio, TX: Author (2008).

135. Ozanian A. Development of the Post-Deployment Health Reassessment. Edwards Air Force Base, CA: US Department of the Air Force (2010).

136. Foa EB, Cashman L, Jaycox L, Perry K. The validation of a self-report measure of posttraumatic stress disorder: The posttraumatic diagnostic scale. Psychol Assess (1997) 9(4):445-51. doi:10.1037/1040-3590.9.4.445

137. Foa EB, Riggs DS, Dancu CV, Rothbaum BO. Reliability and validity of a brief instrument for assessing posttraumatic-stress-disorder. J Trauma Stress (1993) 6(4):459-73. doi:10.1002/jts.2490060405

138. Resnick HS, Falsetti SA, Kilpatrick DG, Freedy JR. Assessment of rape and other civilian trauma-related post-traumatic stress disorder: emphasis on assessment of potentially traumatic events. In: Miller TW, editor. Stressful Life Events. Madison: International Universities Press (1996). p. 231-66.

139. Prins A, Ouimette P, Kimerling R, Cameron RP, Hugelshofer DS, ShawHegwer J, et al. The primary care PTSD screen (PC-PTSD): development and operating characteristics. Prim Care Psychiatry (2003) 9(1):9-14. doi: $10.1185 / 135525703125002360$

140. Weathers F, Litz B, Herman D, Huska J, Keane T. The PTSD checklist (PCL): reliability, validity, and diagnostic utility. Paper Presented at the Annual Convention of the International Society for Traumatic Stress Studies. San Antonio, TX: Boston Veterans Affairs Medical Center (1993).

141. Blanchard EB, JonesAlexander J, Buckley TC, Forneris CA. Psychometric properties of the PTSD checklist (PCL). Behav Res Ther (1996) 34(8):669-73. doi:10.1016/0005-7967(96)00033-2

142. Weathers FW, Litz BT, Huska JA, Keane TM. PCL-C for DSM-IV (11/1/94) National Center for PTSD - Behavioral Science Division. Boston Veterans Affairs Medical Center (1994).
143. Weathers L, Huska K. PTSD Checklist Civilian Version (PCL) PCL-M for DSM-IV (11/1/94) National Center for PTSD - Behavioral Science Division. Boston Veterans Affairs Medical Center (1994).

144. Weathers FW, Litz BT, Huska JA, Keane TM. PCL-S for DSM-IV (11/1/94) National Center for PTSD - Behavioral Science Division. (1994).

145. Watson CG, Juba MP, Manifold V, Kucala T, Anderson PE. The PTSD interview: rationale, description, reliability, and concurrent validity of a DSM-III based technique. J Clin Psychol (1991) 47:179-88. doi:10.1002/10974679(199103)47:2<179::AID-JCLP2270470202>3.0.CO;2-P

146. Hovens JE, Vanderploeg HM, Bramsen I, Klaarenbeek MTA, Schreuder JN, Rivero VV. The development of the self-rating inventory for posttraumatic-stress-disorder. Acta Psychiatr Scand (1994) 90(3):172-83. doi:10.111 1/j.1600-0447.1994.tb01574.x

147. Solomon Z, Benbenishty R, Neria Y, Abramowitz M, Ginzburg K, Ohry A. Assessment of PTSD - validation of the revised PTSD inventory. Isr J Psychiatry Relat Sci (1993) 30(2):110-5.

148. Hendrix CC, Anelli LM, Gibbs JP, Fournier DG. Validation of the Purdue Posttraumatic Stress Scale on a sample of Vietnam veterans. J Trauma Stress (1994) 7(2):311-8. doi:10.1002/jts.2490070211

149. Davidson JRT, Kudler HR, Smith RD. Assessment and Pharmacotherapy of Posttraumatic Stress Disorder. Washington, DC: American Psychiatric Press (1990).

150. Briere J. Trauma Symptom Inventory Professional Manual. Odessa: Psychological Assessment Resources (1995).

151. Alderfer BS, Arciniegas DB, Silver JM. Treatment of depression following traumatic brain injury. J Head Trauma Rehabil (2005) 20(6):544-62. doi:10.1097/00001199-200511000-00006

152. Seel RT, Kreutzer JS. Depression assessment after traumatic brain injury: an empirically based classification method. Arch Phys Med Rehabil (2003) 84(11):1621-8. doi:10.1053/s0003-9993(03)00270-3

153. Ouellet MC, Morin CM, Lavoie A. Volunteer work and psychological health following traumatic brain injury. J Head Trauma Rehabil (2009) 24(4):262-71. doi:10.1097/HTR.0b013e3181a68b73

154. Rapoport MJ, Kiss A, Feinstein A. The impact of major depression on outcome following mild-to-moderate traumatic brain injury in older adults. J Affect Disord (2006) 92(2-3):273-6. doi:10.1016/j.jad.2006.05.022

155. Barnes SM, Walter KH, Chard KM. Does a history of mild traumatic brain injury increase suicide risk in veterans with PTSD? Rehabil Psychol (2012) 57(1):18-26. doi:10.1037/a0027007

156. Whelan-Goodinson R, Ponsford JL, Schonberger M, Johnston L. Predictors of psychiatric disorders following traumatic brain injury. J Head Trauma Rehabil (2010) 25(5):320-9. doi:10.1097/HTR.0b013e3181c8f8e7

157. Zgaljardic DJ, Seale GS, Schaefer LA, Temple RO, Foreman J, Elliott TR. Psychiatric disease and post-acute traumatic brain injury. J Neurotrauma (2015) 32(23):1911-25. doi:10.1089/neu.2014.3569

158. Jorge RE, Robinson RG, Arndt $\mathrm{S}$. Are there symptoms that are specific for depressed mood in patients with traumatic brain injury. J Nerv Ment Dis (1993) 181(2):91-9. doi:10.1097/00005053-199302000-00004

159. O’Donnell ML, Creamer M, Bryant RA, Schnyder U, Shalev A. Posttraumatic disorders following injury: an empirical and methodological review. Clin Psychol Rev (2003) 23(4):587-603. doi:10.1016/s0272-7358(03) 00036-9

160. Sumpter RE, McMillan TM. Misdiagnosis of post-traumatic stress disorder following severe traumatic brain injury. Br J Psychiatry (2005) 186:423-6. doi:10.1192/bjp.186.5.423

161. Hoge CW, McGurk D, Thomas JL, Cox AL, Engel CC, Castro CA. Mild traumatic brain injury in US Soldiers returning from Iraq. N Engl J Med (2008) 358(5):453-63. doi:10.1056/NEJMoa072972

162. O’Donnell ML, Creamer M, Pattison P, Atkin C. Psychiatric morbidity following injury. Am J Psychiatry (2004) 161(3):507-14. doi:10.1176/appi. ajp.161.3.507

163. Ommaya AK, Salazar AM, Dannenberg AL, Chervinsky AB, Schwab $\mathrm{K}$. Outcome after traumatic brain injury in the US military medical system. J Trauma-Injury Infect Crit Care (1996) 41(6):972-5. doi:10.1097/00005373-199612000-00005

164. Pietrzak RH, Johnson DC, Goldstein MB, Malley JC, Southwick SM. Posttraumatic stress disorder mediates the relationship between mild traumatic brain injury and health and psychosocial functioning in veterans 
of operations enduring freedom and Iraqi freedom. J Nerv Ment Dis (2009) 197(10):748-53. doi:10.1097/NMD.0b013e3181b97a75

165. Weathers FW, Keane TM, Davidson JRT. Clinician-administered PTSD scale: a review of the first ten years of research. Depress Anxiety (2001) 13(3):132-56. doi:10.1002/da.1029

166. Polusny MA, Kehle SM, Nelson NW, Erbes CR, Arbisi PA, Thuras P. Longitudinal effects of mild traumatic brain injury and posttraumatic stress disorder comorbidity on postdeployment outcomes in national guard soldiers deployed to Iraq. Arch Gen Psychiatry (2011) 68(1):79-89. doi:10.1001/ archgenpsychiatry.2010.172

167. Sharp TJ, Harvey AG. Chronic pain and posttraumatic stress disorder: mutual maintenance? Clin Psychol Rev (2001) 21(6):857-77. doi:10.1016/ s0272-7358(00)00071-4

168. Harmon KG, Drezner J, Gammons M, Guskiewicz K, Halstead M, Herring S, et al. American medical society for sports medicine position statement: concussion in sport. Clin J Sport Med (2013) 23(1):1-18. doi:10.1097/ JSM.0b013e31827f5f93

169. Biver F, Goldman S, Delvenne V, Luxen A, Demaertelaer V, Hubain P, et al. Frontal and parietal metabolic disturbances in unipolar depression. Biol Psychiatry (1994) 36(6):381-8. doi:10.1016/0006-3223(94)91213-0

170. Ursano RJ, Fullerton CS, Epstein RS, Crowley B, Kao TC, Vance K, et al. Acute and chronic posttraumatic stress disorder in motor vehicle accident victims. Am J Psychiatry (1999) 156(4):589-95.

171. Kupchik M, Strous RD, Erez R, Gonen N, Weizman A, Spivak B. Demographic and clinical characteristics of motor vehicle accident victims in the community general health outpatient clinic: a comparison of PTSD and non-PTSD subjects. Depress Anxiety (2007) 24(4):244-50. doi:10.1002/da.20189

172. Donders J, Levitt T. Criterion validity of the neuropsychological assessment battery after traumatic brain injury. Arch Clin Neuropsychol (2012) 27(4):440-5. doi:10.1093/arclin/acs043

173. Merkley TL, Larson MJ, Bigler ED, Good DA, Perlstein WM. Structural and functional changes of the cingulate gyrus following traumatic brain injury: relation to attention and executive skills. JInt Neuropsychol Soc (2013) 19(8):899-910. doi:10.1017/s135561771300074x
174. Chambers RA, Taylor JR, Potenza MN. Developmental neurocircuitry of motivation in adolescence: a critical period of addiction vulnerability. Am J Psychiatry (2003) 160(6):1041-52. doi:10.1176/appi.ajp.160.6.1041

175. Bjork JM, Grant SJ. Does traumatic brain injury increase risk for substance abuse? J Neurotrauma (2009) 26(7):1077-82. doi:10.1089/neu.2008.0849

176. Bryant RA. Disentangling mild traumatic brain injury and stress reactions. N Engl J Med (2008) 358(5):525-7. doi:10.1056/NEJMe078235

177. Bryant RA, Marosszeky JE, Crooks J, Baguley I, Gurka J. Coping style and post-traumatic stress disorder following severe traumatic brain injury. Brain Injury (2000) 14(2):175-80. doi:10.1080/026990500120826

178. Bryant RA, Marosszeky JE, Crooks J, Baguley IJ, Gurka JA. Interaction of posttraumatic stress disorder and chronic pain following traumatic brain injury. JHead Trauma Rehabil (1999) 14(6):588-94. doi:10.1097/00001199-199912000-00007

179. Bryant RA, Marosszeky JE, Crooks J, Baguley IJ, Gurka JA. Posttraumatic stress disorder and psychosocial functioning after severe traumatic brain injury. J Nerv Ment Dis (2001) 189(2):109-13. doi:10.1097/00005053-20010200000006

180. Turnbull SJ, Campbell EA, Swann IJ. Post-traumatic stress disorder symptoms following a head injury: does amnesia for the event influence the development of symptoms? Brain Inj (2001) 15(9):775-85. doi:10.1080/02699050110034334

Conflict of Interest Statement: The authors declare that the research was conducted in the absence of any commercial or financial relationships that could be construed as a potential conflict of interest.

Copyright ( 2016 Zaninotto, Vicentini, Fregni, Rodrigues, Botelho, de Lucia and Paiva. This is an open-access article distributed under the terms of the Creative Commons Attribution License (CC BY). The use, distribution or reproduction in other forums is permitted, provided the original author(s) or licensor are credited and that the original publication in this journal is cited, in accordance with accepted academic practice. No use, distribution or reproduction is permitted which does not comply with these terms. 